Original Research Paper

\title{
Validation of Tools for 3Dof Orbital Dynamics Simulation
}

\author{
Mariana Poderico and Gianfranco Morani \\ On-board Systems and ATM, Italian Aerospace Research Centre (CIRA), Capua, Italy
}

\author{
Article history \\ Received: 30-07-2020 \\ Revised: $22-10-2020$ \\ Accepted: 31-10-2020 \\ Corresponding Author \\ Gianfranco Morani \\ On-board Systems and ATM, \\ Italian Aerospace Research \\ Centre (CIRA), Capua, Italy \\ Email: g.morani@cira.it
}

\begin{abstract}
This paper describes the validation activities of the 3Dof OrbitalSim tool developed by Italian Aerospace Research Centre (CIRA) and capable of simulating the orbital flight dynamics of a generic spacecraft. The primary strategic goals of the Validation Program of the Orbital-Sim tool were to create an autonomous and effective Orbital Simulator tool for CIRA and Italian community needs. This paper then describes the workflow for the processes and results of Verification and Validation $(\mathrm{V} \& \mathrm{~V})$ for the 3DoF Orbital Dynamics simulation tools. Test cases and results demonstrate that the Orbital-Sim tools have passed successfully all the test cases foreseen by the Validation Program.
\end{abstract}

Keywords: Orbital Platform Simulator, LEO Orbit, 3DOF, Verification and Validation

\section{Introduction}

Nowadays the Aerospace community exploits a huge number of flight dynamics available programs as they are very useful to perform various flight dynamics operations (Vallado, 2005).

The availability of multiple flight dynamics programs suggests the need for standardization. In this framework, the problem of evaluating their accuracy must be considered. As a matter of fact, there are many potential error sources which may depend on several reasons, for instance mathematical model simplifications or computational precision as well as modeling inaccuracies (Vallado, 2005).

Orbital-Sim is an Orbital Platforms Simulation tool developed by Italian Aerospace Research Centre. It has the aim of reproducing the orbital dynamics of a generic spacecraft with different level of accuracy. As a matter of fact, it can be used both to model only the translational dynamics (3DoF configuration) but also to reproduce the complete spacecraft dynamics, i.e., the translational and rotational ones (6DoF configuration). The model has been developed in a MATLAB/Simulink ${ }^{\circledR}$ environment and contains all the elements required to reproduce the dynamic behavior of a spacecraft. Therefore, besides the equations of motion, the subsystems having an impact on spacecraft dynamics are also modelled (i.e., Sensors, Actuators and Engine). Furthermore, the external environment in which the spacecraft flight takes place is also reproduced; therefore, all the effects due to gravity, atmospheric drag, solar radiation pressure, third body perturbation, gravity gradient and magnetic field are taken into account.
Orbital Sim has been developed as an effective tool for mission analysis as well as for the design, development and verification of Guidance, Navigation and Control technologies for space missions.

In this study, the validation activities carried out for the 3Dof part of Orbital-Sim simulation tool will be described and presented. All the test cases and the related results will be reported with the aim of showing the accuracy and reliability of the simulation model.

The paper is made of several sections; at the beginning, an overview of the Orbital-Sim tool is given. The subsequent sections describe the structure of the Validation, i.e., approach, methodology, etc. Finally, the test cases are presented and discussed.

\section{Orbital-Sim System Overview}

\section{Three Dof Orbital-Sim}

Orbital-Sim is a 3-degree-of-freedom model, developed in MATLAB/Simulink ${ }^{\circledR}$ environment (ver. R2017a), capable of simulating the orbital flight dynamics of a generic spacecraft.

It is an Orbital Propagator, which executes the integration of inertial acceleration acting on the spacecraft to obtain velocity and position. Initial position and speed values in ECI reference frame are taken from the orbital initial parameters set in the initialization script. The integration is carried out in inertial ECI reference frame.

During the model development, the following general assumptions have been taking into account: 
1. No rotational dynamics are taken into account, i.e., the spacecraft is always in trimmed conditions

2. Aerodynamic side force is considered negligible

3. The spacecraft is assumed to be a single point of mass

The MATLAB/Simulink® 3DoF tool, Orbital-Sim, is made of a Simulink® model and a set of MATLAB scripts and files, mainly used for the configuration and initialization procedure.

Figure 1 reports the highest level of the " $3 \mathrm{DoF}$ Orbital Simulator", which mainly includes the following elements:

- 3DoF Equations of motion integration

- Spacecraft

$\begin{array}{ll}\circ & \text { Sensors } \\ \circ & \text { Engine }\end{array}$

- Environment

○ Gravity

- Atmospheric drag

- Solar radiation pressure

○ Third body perturbation

The environment effects have been implemented according to the models described in (Vallado, 2013) as configurable library blocks, configured in the main script at the initialization.

\section{Verification and Validation Approach}

The primary strategic goals of the Validation activities of the Orbital-Sim tool, presented in this study, were to:
- Create an autonomous Orbital Simulator, to avoid using very expensive tools

- Create an effective tool for CIRA and Italian community needs

On the other hand, the primary technical goals were to:

- Systematically evaluate and validate all models and components of the tool

- Fix all critical system bugs

- Update working specifications that define tool behavior

- Provide good quality end user documentation

- Prepare for system maintenance and further development

\section{V\&V Philosophy}

The Validation program of Orbital-Sim includes the following main activities (Fig. 2): Explore, Document, Test and Debug, as the majority of Verification and Validation programs recommends in the researcher community (Hughes et al., 2014).

The Explore phase includes the exploration and use of system components to determine the current state. Documentation activities include updating working specifications, writing - test cases and procedures and updating documentation. Testing phase includes - performing additional tests to ensure full coverage. These activities are not necessarily sequential but for a given component a few cycles through these activities could be needed.
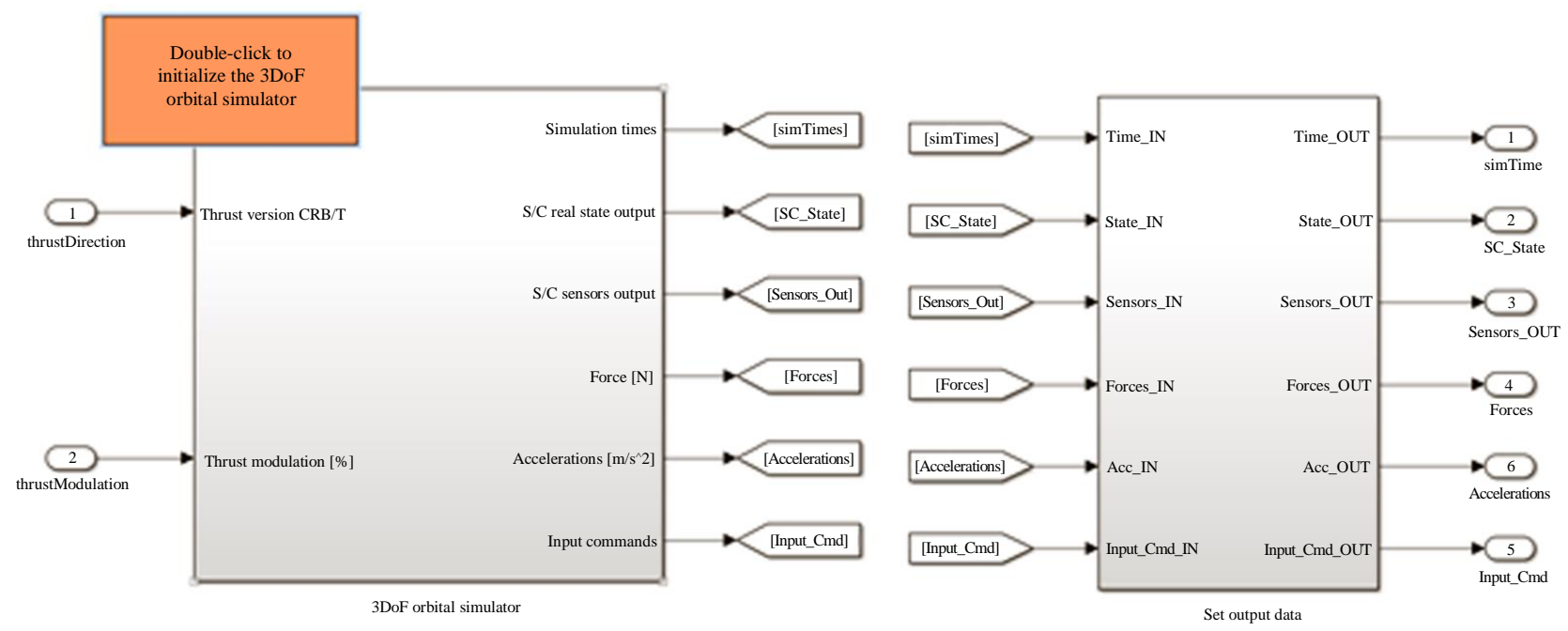

Fig. 1: Orbital-Sim 3DoF simulation model 


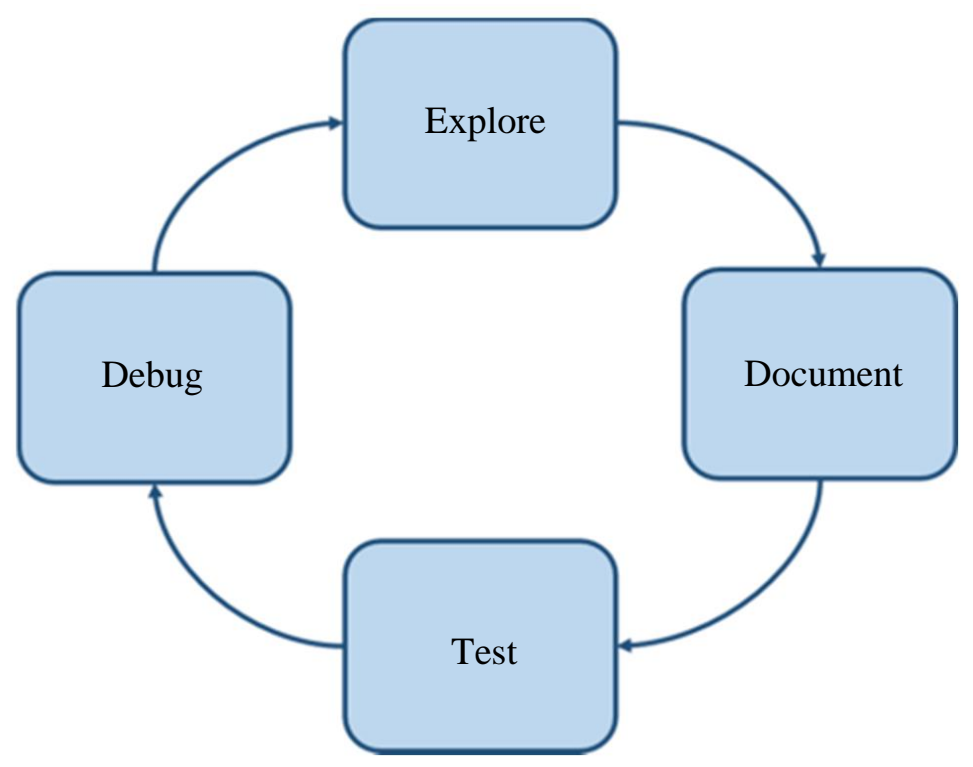

Fig. 2: High level V\&V approach

\section{V\&V Methodology}

The V\&V methodology used to validate the Orbital-Sim tool is the industry standard one. The major goal is to implement test procedures in repeatable and automated test environments to support the testing of the whole simulator tool during development activities.

The primary Test Typologies performed are the following ones (Hughes et al., 2014):

- $\quad$ Numeric Tests: Tests of physical and mathematical model. Numeric tests are performed by comparing output to the external "truth"

- End-to-end tests: Test that solve an end-to-end problem such as orbital maneuvers. These tests are applications of Orbital Simulator to the realworld problems

In order to carry out the Validation plan, system components have been grouped under two categories:

- Dynamics and Models: Numerical models for orbit propagation, coordinate system models, environment effects

- Powered Flight: Numerical models for impulsive and finite maneuvering including tanks and thrusters

\section{$V \& V$ Environment}

The Validation activities have been carried out through a MATALB/Simulink® simulation environment by which automated regression tests have been performed.

In particular, MATLAB scripts implement test procedures (more details are reported in the next section) in order to compare the results of the Orbital Simulator with two benchmarks selected for these validation activities, i.e.:

1. General Mission Analysis Tool (GMAT), version R2018a (NASA 2018a). It is a space mission design software for the design and optimization of missions (anywhere in the solar system) ranging from low Earth orbit to lunar, Libration point and deep space missions

2. Systems Tool Kit (STK), version 9, (AGI, STK, 2020). STK is a physics-based software package from Analytical Graphics, Inc., AGI, that allows engineers and scientists to perform complex analyses of ground, sea, air and space platforms and share results in one integrated environment

It is worth noting that, due the limited licenses of the above benchmark tools, neither GMAT nor STK allowed a complete (i.e., for all the test cases) validation process. Therefore, for some test cases only GMAT has been used, for other cases only STK.

It's worth noting that GMAT propagator itself has been validated (Dove and Hughes, 2007; Hughes et al., 2014) through comparison with STK propagator, which is currently considered the most reliable Orbital Simulation toolkit. 
Table 1: Validation test summary

\begin{tabular}{lll}
\hline Test_Dyn\&Mod_001 & Orbit Propagation and gravity effects & STK \\
Test_Dyn\&Mod_002 & Orbit Propagation and gravity effects & STK \\
Test_Dyn\&Mod_003 & Solar radiation pressure effect & STK \\
Test_Dyn\&Mod_004 & Solar radiation pressure effect & STK \\
Test_Dyn\&Mod_005 & Solar radiation pressure effect \\
Test_Dyn\&Mod_006 & Third body perturbation effect & STK \\
Test_Dyn\&Mod_007 & Third body perturbation effect & STK \\
Test_Dyn\&Mod_008 & Atmospheric drag & STK \\
Test_Dyn\&Mod_009 & Orbit Propagation and environment effects & STK \\
Test_PowFlight_001 & In plane electric orbital maneuver & STK \\
Test_PowFlight_002 & In plane electric orbital maneuver & GMAT \\
Test_PowFlight_003 & In plane chemical orbital maneuver & GMAT \\
Test_PowFlight_004 & In plane chemical orbital maneuver & GMAT \\
Test_PowFlight_005 & In plane chemical orbital maneuver & GMAT \\
Test_PowFlight_006 & In plane chemical orbital maneuver & GMAT \\
Test_PowFlight_007 & Out of plane chemical orbital maneuver & GMAT \\
Test_PowFlight_008 & Out of plane chemical orbital maneuver & GMAT \\
Test_PowFlight_009 & Out of plane chemical orbital maneuver & GMAT \\
Test_PowFlight_010 & Deorbiting maneuver & GMAT \\
\hline
\end{tabular}

\section{V\&V Approach}

As explained earlier, the Validation plan includes a comparison two by two between Orbital-Sim and GMAT and STK. It's worth noting that STK has not been used for the Powered Flight comparison, due to the lack of the license for STK tool that includes the orbital maneuvers.

Table 1 provides a summary of the Validation program. In the following sections, all the test cases will be reported with the results.

\section{Verification and Validation Results}

This section details the results of the test cases reported in Table 1. As explained earlier, the tests have been grouped under two categories, i.e., "Dynamics and Models" and "Powered Flight".

In particular, in the category "Dynamics and Models" all the significant environmental effects for the Low Earth Orbits are included, while for what concerns the category "Powered Flight", some relevant maneuvers have been considered such as in-plane, out-of-plane and De-orbit with both Chemical and Electric Thrust.

Parameters settings for Earth and Sun, applied to all the test cases, are in accordance with Vallado (2013; IERS Conventions, 2010; NASA, 2018b).

Each test description includes the following fields:

- Test id: A label identifying the test

- Objective: Functionality to be tested

- Conditions: Configuration and initialization needed to execute the test

- Test procedures: Description of the procedure step by step to carry out the test
- Test results: Results are reported as RMS error (on given variables)

- Criterion: To evaluate the outcome of a test case, in terms of orbit propagation accuracy, an acceptance matrix was created on the basis of what contained in (Dove and Hughes, 2007) (which considers also the analyses reported in Vallado, 2005). The values in Table 2 were obtained from the above references, applying a conservative scale factor, which depends on the following aspects:

- The values reported in (Dove and Hughes, 2007) are referred to instantaneous values while test cases results are RMS errors. In this respect, assuming an oscillating (quasi) sinusoidal behavior for the error, the applied scale factor is square root of 2

- The values are referred to the norm of position error, while test cases are referred to the error components. Therefore a conservative scale factor equal to square root of 3 has been considered

Furthermore, the obtained results will be also compared with the ones reported in Robson (2018) where an Orbit and Attitude Simulation Environment has been described and validated.

As explained earlier, some tests concern numerical models for impulsive and finite maneuvering including tanks and thrusters, the "Powered Flight" category. In this respect, the following engine models will be considered:

- Electric Thruster model: It has been obtained considering a polynomial function for the thrust and 
mass flow rate (as in GMAT User Guide, 2018). Values of the coefficients are reported in Table 3

- Chemical Thruster model: Thrust and specific impulse are defined as a polynomial function of pressure. As a consequence, the mass flow rate is also polynomial (GMAT User Guide, 2018). In Tables 4 and 5 the parameters of the thruster model are reported

Table 2: Acceptance matrix for position error $(\mathrm{km})$

\begin{tabular}{ll}
\hline Difference in & Acceptable position error $(\mathrm{km})$ \\
\hline Point mass gravity & $<4.082482904639 \mathrm{e}-07$ \\
Non-spherical gravity & $<4.082482904639 \mathrm{e}-07$ \\
Drag & $<8.164965809277 \mathrm{e}-03$ \\
Solar radiation pressure & $<2.449489742783 \mathrm{e}-04$ \\
Third body perturbation & $<2.449489742783 \mathrm{e}-06$ \\
Combined perturbation & $<8.164965809277 \mathrm{e}-03$ \\
\hline
\end{tabular}

Table 3: Electric thruster coefficients

\begin{tabular}{llll}
\hline Mass flow & & & \\
coefficients & Values & Thrust coefficients & Values \\
\hline Electric prop Cm1 & -0.004776 & Electric Prop Ct1 & -5.19082 \\
Electric prop Cm2 & 0.05717 & Electric Prop Ct2 & 2.96519 \\
Electric prop Cm3 & -0.09956 & Electric Prop Ct3 & -14.4789 \\
Electric prop Cm4 & 0.03211 & Electric Prop Ct4 & 54.05382 \\
Electric prop Cm5 & 2.13781 & Electric Prop Ct5 & 0.00100092 \\
\hline
\end{tabular}

Table 4: Chemical thruster-thrust coefficients

\begin{tabular}{lll}
\hline Thrust coefficients & Values & Units \\
\hline C1 & 270 & $\mathrm{~N}$ \\
C 2 & 0.01 & $\mathrm{~N} / \mathrm{kPa}$ \\
C 3 & 0 & $\mathrm{~N} / \mathrm{kPa} 2$ \\
C 4 & 0 & $\mathrm{~N} / \mathrm{kPaC} 5$ \\
C 5 & 0 & None \\
C 6 & 0 & $\mathrm{~N} / \mathrm{kPaC} 7$ \\
C 7 & 0 & None \\
C 8 & 0 & $\mathrm{~N} / \mathrm{kPaC} 9$ \\
C 9 & 0 & None \\
C 10 & 0 & $\mathrm{~N}$ \\
C 11 & 0 & none \\
C 12 & 0 & $1 / \mathrm{kPa}$ \\
C 13 & 0 & none \\
C 14 & 0 & $1 / \mathrm{kPa}$ \\
\hline
\end{tabular}

Table 5: Chemical thruster - specific impulse coefficients

\begin{tabular}{lll}
\hline Thrust coefficients & Values & Units \\
\hline K1 & 280 & $\mathrm{~s}$ \\
K2 & 0.01 & $\mathrm{~s} / \mathrm{kPa}$ \\
K3 & 0 & $\mathrm{~s} / \mathrm{kPa} 2$ \\
K4 & 0 & $\mathrm{~s} / \mathrm{kPak} 5$ \\
K5 & 0 & None \\
K6 & 0 & $\mathrm{~s} / \mathrm{kPak} 7$ \\
K7 & 0 & None \\
K8 & 0 & $\mathrm{~s} / \mathrm{kPak} 9$ \\
K9 & 0 & None \\
K10 & 0 & $\mathrm{~s}$ \\
K11 & 0 & none \\
K12 & 0 & $1 / \mathrm{kPa}$ \\
K13 & 0 & none \\
K14 & 0 & $1 / \mathrm{kPa}$ \\
\hline
\end{tabular}

Test Cases Dyn\&Mod_001-002 Orbit Propagation and Gravity Effects

Objective: Validation of orbital dynamics at different altitudes with different Gravity models.

Conditions: Propagation of circular orbits at altitudes of $300 \mathrm{~km}$ with different gravity models. The configuration parameters are reported in Table 6.

Test results: Results show that the RMS position error is of the order of $\mathrm{cm}$ on the $Z$ component wrt STK one (Table 7).

Criterion: RMS errors < 4.082482904639 e-07 (see Table 2). Tests PASSED.

\section{Test Cases Dyn\&Mod_003-005 Solar Radiation Pressure Effect}

Objective: Validation of the Environment: Solar Radiation Pressure effect (SRP).

Conditions: Propagation of circular orbits at altitudes of $300,10000 \mathrm{~km}$ with the solar radiation pressure effect. Notice that, in Orbital-Sim, the effect of the shadow of the Earth on the satellite has been implemented. In particular, cylindrical and conical shadows have been considered. The configuration parameters are reported in Table 8 .

Test results: Results show that the RMS position error is of the order of mm wrt STK one (Table 9).

Criterion: RMS errors < 2.449489742783 e-04 (Table 2). Tests PASSED.

\section{Test Cases Dyn\&Mod_006-007 Third Body Perturbation Effect}

Objective: Validation of the Environment: Third Body perturbation effect (3B).

Conditions: Propagation of circular orbits at altitudes of $300 \mathrm{Km}$ with the third body perturbation effect. It's worth noting that the third body perturbations of Moon and Sun have been considered. The configuration parameters are reported in Table 10.

Test results: Results show that the RMS position error is of the order of cm-mm wrt STK one (Table 11).

Criterion: RMS errors < 2.449489742783e-06 (Table 2). Tests PASSED.

Table 6: Initialization and configuration

\begin{tabular}{ll}
\hline Altitude $[\mathrm{km}]$ & 300 \\
Gravity model & $\mathrm{J} 2, \mathrm{~J} 3$ \\
Environment effects & Neglected \\
Satellite epoch & $01 / 06 / 2010$ \\
Satellite mass $[\mathrm{kg}]$ & 250 \\
Reference frame & ICFR \\
Earth model & WGS84-EGM2008 \\
Numerical Solver & Fixed step, Runge-Kutta 4 \\
\hline
\end{tabular}




\section{Test Cases Dyn\&Mod_008 Atmospheric Drag}

Objective: Validation of the Environment: Atmospheric drag.

Conditions: Propagation of circular orbits at altitudes of $300 \mathrm{~km}$ with the effect of the atmospheric drag effect. The model of atmosphere used in Orbital Sim is Nrmlsise00 (NRLMSISE, 2000). The configuration parameters are reported in Table 12.
Test results: Results show that the RMS position error is of the order of $\mathrm{m}$ wrt STK one (Table 13).

It's worth noting that this error is higher than the ones obtained with the other environment effects. This may be due to some differences and/or inaccuracies in the atmospheric models used for the drag force computation which may lead to differences on the propagated orbit (Vallado and Finkleman, 2014).

Criterion: RMS errors < 8.164965809277 e-03 (Table 2). Tests PASSED.

Table 7: Test cases Dyn\&Mod_001-002 STK results (ICRF position components RMS error)

\begin{tabular}{llll}
\hline Orbit Altitudes $[\mathrm{km}]$ & X RMS error & YRMS error & Z RMS error \\
\hline 300 with J2 & $9.731937158848 \mathrm{e}-07$ & $9.824023496630 \mathrm{e}-07$ & $1.107676797431 \mathrm{e}-04$ \\
300 with J3 & $1.189376191472 \mathrm{e}-06$ & $1.272762825925 \mathrm{e}-06$ & $1.104716302140 \mathrm{e}-04$ \\
\hline
\end{tabular}

Table 8: Initialization and Configuration

\begin{tabular}{ll}
\hline Altitude $[\mathrm{km}]$ & 300,10000 \\
Gravity model & Spherical, J2 \\
Environment effects & Solar radiation pressure with cylindrical/conical shadow \\
Satellite epoch & $01 / 06 / 2010$ \\
Satellite mass $[\mathrm{kg}]$ & 250 \\
Reference frame & ICFR \\
Earth model & WGS84-EGM 2008 \\
Numerical Solver & Fixed step, Runge-Kutta 4 \\
\hline
\end{tabular}

Table 9: Test cases Dyn\&Mod_003-005 STK results (ICRF position components RMS error)

\begin{tabular}{llll}
\hline Orbit Altitudes $[\mathrm{km}]$ & X RMS error & Y RMS error & Z RMS error \\
\hline 300, spher, SRP cil & $6.317587478296 \mathrm{e}-06$ & $6.017363999036 \mathrm{e}-06$ & $2.122786087498 \mathrm{e}-07$ \\
300, spher, SRP con & $6.256720082275 \mathrm{e}-06$ & $5.996560754663 \mathrm{e}-06$ & $2.108647197596 \mathrm{e}-07$ \\
10000, spher, SRP cil & $7.283507429331 \mathrm{e}-06$ & $7.07469520217 \mathrm{e}-06$ & $1.111607603126 \mathrm{e}-06$ \\
10000, spher, SRP con & $6.734326806202 \mathrm{e}-06$ & $6.642108840629 \mathrm{e}-06$ & $1.026466583987 \mathrm{e}-06$ \\
\hline
\end{tabular}

Table 10: Initialization and configuration

\begin{tabular}{ll}
\hline Altitude $[\mathrm{km}]$ & 300 \\
Gravity model & Spherical, J2 \\
Environment effects & Third body perturbation \\
Satellite epoch & $01 / 06 / 2010$ \\
Satellite mass $[\mathrm{kg}]$ & 250 \\
Reference frame & ICFR \\
Earth model & WGS84-EGM 2008 \\
Numerical Solver & Fixed step, Runge-Kutta 4 \\
\hline
\end{tabular}

Table 11: Test cases Dyn\&Mod_006-007 STK results (ICRF position components RMS error)

\begin{tabular}{llll}
\hline Orbit Altitudes [km] & X RMS error & Y RMS error & Z RMS error \\
\hline 300, spherical, 3B & $1.697755701269 \mathrm{e}-06$ & $1.656983191464 \mathrm{e}-06$ & $2.873346803037 \mathrm{e}-07$ \\
\hline
\end{tabular}

Table 12: Initialization and Configuration.

\begin{tabular}{ll}
\hline Altitude $[\mathrm{km}]$ & 300 \\
Gravity model & Spherical \\
Environment effects & Atmospheric drag \\
Satellite epoch & $01 / 06 / 2010$ \\
Satellite mass $[\mathrm{kg}]$ & 250 \\
Reference frame & ICFR \\
Earth model & WGS84-EGM 2008 \\
Numerical Solver & Fixed step, Runge-Kutta 4 \\
\hline
\end{tabular}

Table 13: Test cases Dyn\&Mod_008 STK results (ICRF position components RMS error)

\begin{tabular}{llll}
\hline Orbit Altitudes [km] & X RMS error & Y RMS error & Z RMS error \\
\hline 300, spherical, NRLMSISE00 & $1.565158852334 \mathrm{e}-3$ & $2.747121968042 \mathrm{e}-3$ & $1.001938030072 \mathrm{e}-06$ \\
\hline
\end{tabular}




\section{Test Cases Dyn\&Mod_009 Orbit Propagation and Environment Effects}

Objective: Validation of Orbit Propagation and environment effects.

Conditions: Propagation of circular orbits at altitudes of $300 \mathrm{~km}$ with the enabling all the above effects put together. The model of atmosphere used in Orbital Sim is NRMLSISE00. The configuration parameters are reported in Table 14.

Test results: Results show that the RMS position error is of the order of $\mathrm{m}$ wrt STK one (Table 15).

Criterion: RMS errors < 8.164965809277e-03 (Table 2). Tests PASSED.

\section{Test Cases PowFlight_001-002 In plane Electric Orbital Maneuver}

Objective: Validation of Powered Flight: In plane electric orbital maneuver.

Conditions: Propagation of circular orbits at altitudes of $300 \mathrm{~km}$ with spherical gravity model. At time $\mathrm{t}=5430$ $\mathrm{sec}$, the electric thruster has been switched on in order to execute an in plane maneuver. The configuration parameters are reported in Table 16.

Test results: Results show that the RMS position error is of the order of meters wrt GMAT for the in plane components and up to millimeters for the out of plane component (Table 17).

Criterion: N/A.

\section{Test Cases PowFlight_003-006 In plane Chemical Orbital Maneuver}

Objective: Validation of Powered flight: in plane chemical orbital maneuvers.

Conditions: Propagation of circular orbits at altitudes of 300 with spherical gravity model. At time $\mathrm{t}=5430 \mathrm{sec}$, the chemical thruster has been switched on in order to execute an in plane maneuver. The configuration parameters are reported in Table 18.

Test results: Results show that the RMS position error wrt GMAT goes from the meters to dozens of meters depending on the maximum thrust and the thrust duration (Table 19).

Criterion: N/A.

Test Cases PowFlight_007-009 Out of plane Chemical Orbital Maneuver

Objective: Validation of Powered flight: Out of plane chemical orbital maneuvers.

Conditions: Propagation of circular orbits at altitudes of 300 with spherical gravity model. At time $\mathrm{t}=5430 \mathrm{sec}$ the chemical thruster has been switched on in order execute out an out of plane maneuver. The configuration parameters are reported in Table 20.

Test results: Results show that the RMS position error is of the order of hundreds of meter wrt GMAT one for the $270 \mathrm{~N}$ case and dozens of meter for the $27 \mathrm{~N}$ case (Table 21).

Criterion: N/A.

Table 14: Initialization and configuration

\begin{tabular}{ll}
\hline Altitude $[\mathrm{km}]$ & 300 \\
Gravity model & Spherical \\
Environment effects & Solar radiation pressure Third body perturbation Atmospheric drag \\
Satellite epoch & $01 / 06 / 2010$ \\
Satellite mass $[\mathrm{kg}]$ & 250 \\
Reference frame & ICFR \\
Earth model & WGS84-EGM 2008 \\
Numerical solver & Fixed step, Runge-Kutta 4 \\
\hline
\end{tabular}

Table 15: Test cases Dyn\&Mod_009 STK results (ICRF position components RMS error)

\begin{tabular}{llll}
\hline Orbit Altitudes [km] & X RMS error & Y RMS error & Z RMS error \\
\hline 300, J2, SRP, 3B, NRMLSISE00 & $4.457323850167 \mathrm{e}-03$ & $5.631685877329 \mathrm{e}-03$ & $4.430447618301 \mathrm{e}-05$ \\
\hline
\end{tabular}

Table 16: Initialization and configuration

\begin{tabular}{|c|c|}
\hline Altitude $[\mathrm{km}]$ & 300 \\
\hline Gravity model & Spherical \\
\hline Environment effects & Neglected \\
\hline Electric Thruster & 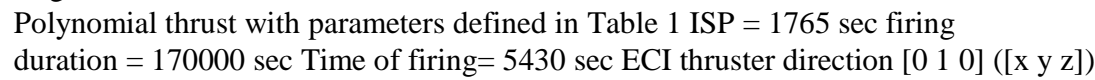 \\
\hline Satellite epoch & $01 / 06 / 2010$ \\
\hline Satellite mass $[\mathrm{kg}]$ & 250 \\
\hline Reference frame & ICFR \\
\hline Earth model & WGS84-EGM 2008 \\
\hline Numerical solver & Fixed step, Runge-Kutta 4 \\
\hline
\end{tabular}


Table 17: Test cases PowFlight_001-002 GMAT results (ICRF position components RMS error)

\begin{tabular}{llll}
\hline Orbit Altitudes [km] & X RMS error & Y RMS error & Z RMS error \\
\hline 300, spherical, polynomial thrust & $1.860524260727 \mathrm{e}-03$ & $1.896805578657 \mathrm{e}-03$ & $5.662283546193 \mathrm{e}-07$ \\
\hline
\end{tabular}

Table 18: Initialization and Configuration

\begin{tabular}{ll}
\hline Altitude $[\mathrm{km}]$ & 300 \\
Gravity model & Spherical \\
Environment effects & Neglected \\
Chemical Thruster & $\begin{array}{l}\text { 1. Polynomial thrust with: } \\
\text { firing duration }=20 \mathrm{sec}\end{array}$ \\
& $\begin{array}{l}\text { 2. Polynomial thrust with: } \\
\text { s/KPa firing duration }=\end{array}$ \\
& 3. Polynomial thrust with: \\
& firing duration = 200 sec \\
& 4. Polynomial thrust with: \\
& s/KPa firing duration $=$ \\
& 01/06/2010 \\
Satellite epoch & 250 \\
Satellite mass $[\mathrm{kg}]$ & ICFR \\
Reference frame & WGS84-EGM 2008 \\
Earth model & Fixed step, Runge-Kutta 4 \\
Numerical Solver &
\end{tabular}

Table 19: Test cases PowFlight_003-006 GMAT results (ICRF position components RMS error)

\begin{tabular}{llll}
\hline Orbit Altitudes $[\mathrm{km}]$ & X RMS error & Y RMS error & Z RMS error \\
\hline 300, spherical, polynomial thrust, $\mathrm{C} 1=270$, fir $=20 \mathrm{sec}$ & $1.516022719385 \mathrm{e}-01$ & $1.360756365133 \mathrm{e}-01$ & $8.025890423907 \mathrm{e}-07$ \\
300, spherical, polynomial thrust, $\mathrm{C} 1=27, \mathrm{fir}=20 \mathrm{sec}$ & $1.454481335788 \mathrm{e}-03$ & $3.487257449750 \mathrm{e}-03$ & $2.550305761330 \mathrm{e}-07$ \\
300, spherical polynomial thrust, $\mathrm{C} 1=270, \mathrm{fir}=200 \mathrm{sec}$ & $2.066235658546 \mathrm{e}-01$ & $3.059260493165 \mathrm{e}-01$ & $6.493924391780 \mathrm{e}-06$ \\
300, spherical polynomial thrust, $\mathrm{C} 1=27, \mathrm{fir}=200 \mathrm{sec}$ & $2.102005231742 \mathrm{e}-02$ & $3.733377336443 \mathrm{e}-02$ & $7.866869210687 \mathrm{e}-07$ \\
\hline
\end{tabular}

Table 20: Initialization and configuration

Altitude [km] 300

Gravity model Spherical

Environment effects Neglected

Chemical Thruster

1. Polynomial thrust with: $\mathrm{C} 1=270 \mathrm{~N}$ (max thrust), $\mathrm{C} 2=0.01 \mathrm{~N} / \mathrm{KPa} \mathrm{K} 1=280 \mathrm{~s}$ (ISP), $\mathrm{K} 2=0.01$

$\mathrm{s} / \mathrm{KPa}$ firing duration $=200 \mathrm{sec}$ Time of firing $=5430 \mathrm{sec}$ ECI thruster direction $\left[\begin{array}{lll}0 & 0 & 1\end{array}\right]([\mathrm{x} \mathrm{y} \mathrm{z}])$

2. Polynomial thrust with: $\mathrm{C} 1=27 \mathrm{~N}$ (max thrust), $\mathrm{C} 2=0.001 \mathrm{~N} / \mathrm{KPa} \mathrm{K} 1=280 \mathrm{~s}$ (ISP), $\mathrm{K} 2=0.01$

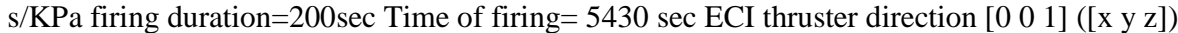

Satellite epoch

Satellite mass $[\mathrm{kg}]$

$01 / 06 / 2010$

Reference frame

Earth model WGS84-EGM 2008

Numerical Solver $\quad$ Fixed step, Runge-Kutta 4

Table 21: Test cases PowFlight_007-009 GMAT results (ICRF position components RMS error)

\begin{tabular}{llll}
\hline Orbit Altitudes [km] & X RMS error & Y RMS error & Z RMS error \\
\hline 300, Spherical gravity Polynomial thrust, $\mathrm{C} 1=270$, fir $=200 \mathrm{sec}$ & $1.100000000000 \mathrm{e}-02$ & $1.490000000000 \mathrm{e}-02$ & $1.419000000000 \mathrm{e}-01$ \\
300, Spherical gravity Polynomial thrust, $\mathrm{C} 1=27$, fir $=200 \mathrm{sec}$ & $4.358993920808 \mathrm{e}-05$ & $1.462113517434 \mathrm{e}-03$ & $1.502941504840 \mathrm{e}-02$ \\
\hline
\end{tabular}

Table 22: Initialization and configuration

\begin{tabular}{ll}
\hline Altitude $[\mathrm{km}]$ & 300 \\
Gravity model & Spherical \\
Environment effects & Neglected \\
Electric Thruster & Constant thrust with: C1 = 750 N (max thrust), C2 =0 N/KPa K1 = 280 s (ISP), K2 =0 s/KPa \\
& firing duration $=45 \mathrm{sec}$ Time of firing = 5430 sec ECI thruster direction $[0-10]([\mathrm{x} \mathrm{y} \mathrm{z])}$ \\
Satellite epoch & $01 / 06 / 2010$ \\
Satellite mass $[\mathrm{kg}]$ & 250 \\
Reference frame & ICFR \\
Earth model & WGS84-EGM 2008 \\
Numerical Solver & Fixed step, Runge-Kutta 4 \\
\hline
\end{tabular}

Table 23: Test cases PowFlight_003-006 GMAT results (ICRF position components RMS error)

\begin{tabular}{llll}
\hline Orbit Altitudes [km] & X RMS error & Y RMS error & Z RMS error \\
\hline 400, spherical, constant thrust, $\mathrm{C} 1=750$, fir $=45 \mathrm{sec}$ & $1.090918804118 \mathrm{e}-02$ & $6.484779794648 \mathrm{e}-02$ & $1.354801415772 \mathrm{e}-06$ \\
\hline
\end{tabular}




\section{Test cases PowFlight_0010 Deorbiting Maneuver}

Objective: Validation of Powered flight: Deorbiting maneuver.

Conditions: Propagation of circular orbits at altitudes of 400 with the execution of a deorbiting maneuver at $\mathrm{t}=5430 \mathrm{sec}$. The configuration parameters are reported in Table 22.

Test results: Results show that the RMS position error wrt GMAT one is of the order of dozens of meters (Table 23).

Criterion: N/A.

\section{Conclusion}

The paper presents the results of validation the activities for the 3DoF part of the Orbital-Sim tool, developed by Italian Aerospace Research Centre. These activities had the goal of comparing physical and mathematical models output to external "truth", represented by the results obtained with a benchmark simulation tool.

The Validation program considered two main categories of testing cases:

- Testing of Dynamics and models, which includes tests of all the models used to describe orbit propagation and environment effects

- Testing of Powered flight, which concerns all the test cases related to the validation of maneuvers: Electrical and chemical maneuver, deorbiting and Hohmann Transfer

The main benchmark used for the Validation program are GMAT and STK, both identified as the best ones for flexibility and functionalities presented.

Test cases and results demonstrate that the OrbitalSim tools have passed successfully all the Validation Program test cases falling under the category named "Dynamics and Models". Furthermore, the results are even better (in terms of comparison with the reference benchmark) than the ones reported in Robson (2018) where an Orbital Propagator has been validated with similar simulation scenarios.

With reference to the Powered flight test cases, for all the reasons explained in the paper, there are no sufficient elements to evaluate the test outcomes.

Therefore, results showed that all tests are passed as they satisfy the accuracy requirements defined at the beginning of validation process.

\section{Acknowledgement}

The authors would like to thank Daniele Romagnoli and Ferdinando Montemari for their major contribution to the development of 3DoF and 6DoF Orbital-Sim tools.

\section{Author's Contributions}

All the authors contributed to the methodological aspects and to the definition of the Validation process as well as to the paper preparation.

M. Poderico was also involved in the simulation setup and in the execution of the numerical verifications.

\section{Ethics}

This article is original and contains unpublished material. The corresponding author confirms that all of the other authors have read and approved the manuscript and no ethical issues involved.

\section{References}

AGI, STK. (2020). Ansys, AGI Sign Definitive Acquisition Agreement. AGI, STK. http://www.agi.com/home

Dove, E., \& Hughes, S. (2007). General Mission Analysis Tool (GMAT) Acceptance Test Plan [Draft].

GMAT. (2018). GMAT User Guide. http://gmat.sourceforge.net/docs/R2018a/help-a4.pdf

Hughes, S. P., Qureshi, R. H., Cooley, S. D., \& Parker, J. J. (2014). Verification and validation of the general mission analysis tool (GMAT). In AIAA/AAS astrodynamics specialist conference (p. 4151).

IERS Conventions. (2010). IERS ConventionsTechnical note N. 36, 2010.

NASA. (2018a). General Mission Analysis Tool (GMAT) Version R2018a. NASA. https://software.nasa.gov/software/GSC-18094-1

NASA. (2018b). Sun Fact Sheet. NASA. https://nssdc.gsfc.nasa.gov/planetary/factsheet/sunfa ct.html

NRLMSISE. (2000). NRLMSISE-00 Atmosphere Model. https://ccmc.gsfc.nasa.gov/modelweb/models/nrlmsi se00.php

Robson, C. (2018). The Design and Validation of a Spacecraft Orbit and Attitude Simulation Environment in MATLAB/Simulink.

Vallado, D. A. (2005, January). An analysis of state vector propagation using differing flight dynamics programs. In Paper AAS 05-199 presented at the AAS/AIAA Space Flight Mechanics Conference. Copper Mountain, $\mathrm{CO}$.

Vallado, D. A. (2013). Fundamentals of Astrodynamics and Applications. Springer Science \& Business Media.

Vallado, D. A., \& Finkleman, D. (2014). A critical assessment of satellite drag and atmospheric density modeling. Acta Astronautica, 95, 141-165. 